\title{
ILCEA
}

Revue de l'Institut des langues et cultures

d'Europe, Amérique, Afrique, Asie et Australie

$38 \mid 2020$

Chant et nation : de la culture populaire à la culture savante

\section{Harmonie verlainienne dans la poésie de Nicolas Minsky}

Verlainian Harmony in the Poetry of Nicolas Minsky

Daria Kuntsevich

\section{OpenEdition}

Journals

Édition électronique

URL : http://journals.openedition.org/ilcea/9758

DOI : $10.4000 /$ ilcea.9758

ISSN : 2101-0609

Éditeur

UGA Éditions/Université Grenoble Alpes

Édition imprimée

ISBN : 978-2-37747-172-0

ISSN : 1639-6073

Référence électronique

Daria Kuntsevich, «Harmonie verlainienne dans la poésie de Nicolas Minsky », ILCEA [En ligne], 38|

2020, mis en ligne le 31 janvier 2020, consulté le 31 janvier 2020. URL : http://

journals.openedition.org/ilcea/9758; DOI : 10.4000/ilcea.9758

Ce document a été généré automatiquement le 31 janvier 2020.

(C) ILCEA 


\title{
Harmonie verlainienne dans la poésie de Nicolas Minsky
}

Verlainian Harmony in the Poetry of Nicolas Minsky

\author{
Daria Kuntsevich
}

\section{Introduction}

1 Le symbolisme, apparu en France dans les années $1870^{1}$, est assimilé en Russie dans les années $1890^{2}$ et y devient un mode de vie et une forme de pensée. En France, Charles Baudelaire, Paul Verlaine, Arthur Rimbaud et Stéphane Mallarmé sont les premiers poètes à tenter de rompre avec les stéréotypes et les clichés de leurs prédécesseurs : les naturalistes, les réalistes, l'école du Parnasse et du romantisme. En Russie, les principes poétiques essentiels du symbolisme sont apparus non seulement en raison de la réaction contre les mouvements littéraires précédents, mais aussi grâce à l'importance des échanges culturels avec la France.

2 La poésie de cette époque en France autant qu'en Russie, globalement, est vue comme un chemin vers une compréhension synthétisante du monde. Dans cette perspective, l'art n'acquiert son intégrité qu'à travers l'unité de la musique, de la poésie, de la peinture. Les éléments divers et séparés deviennent reliés, autrement dit, harmonieux. Les auteurs renouvellent les formes poétiques pour qu'elles deviennent le gage de l'authenticité d'une parole poétique exprimant réflexions et sensations de la vie et de la mort.

3 Selon Verlaine, l'organisation romantique ou parnassienne des vers ne peut pas transmettre les nuances, suggérer les sensations, l'angoisse, le malaise, les rêves, tout ce qui est l'objet de la poésie contemporaine. Dans «l'Art poétique » du recueil Jadis et naguère (1871-1873, qui n'a paru qu'en 1884), Verlaine exprime la priorité de la musique sur le sens :

De la musique encore et toujours !

Que ton vers soit la chose envolée 
Qu'on sent qui fuit d'une âme en allée

Vers d'autres cieux à d'autres amours. musique : la fonction sémantique du mot est affaiblie, le sens fusionne avec la sonorité musicale. Son modèle poétique s'articule sur deux axes de coordonnées: le monde matériel et le monde de son âme. Le vent de la «Chanson d'automne » va et vient entre ces deux mondes et l'emporte "deçà » et "delà ». Le héros lyrique de Verlaine se retrouve entre deux mondes. Des assonances et des sons nasaux (qui n'existent pas dans la langue russe moderne) servent à construire le troisième axe - un axe sonore. Le mot devient musical et fusionne au rythme de l'âme, ce qui sert à exprimer son état d'âme. Le recueil Poèmes saturniens, dans lequel la «Chanson d'automne» est publiée, marque la naissance de « la chanson grise » de Verlaine. Le poète cherche à remplir sa poésie de la musique et le lecteur a pour tâche d'écouter l'harmonie du texte. Ses principes poétiques deviennent un des fondements de l'art symboliste non seulement en France mais aussi en Russie.

5 En Russie, les poèmes de Verlaine sont le plus souvent traduits par Valéry Brioussov et Fiodor Sologoub. En 1894, paraît le recueil Romances sans paroles (Verlaine, 1894), pour la première fois traduit en russe par Brioussov. En 1904, Nicolas Minsky traduit ${ }^{3}$ également quelques poèmes du recueil Romances sans paroles et Poèmes saturniens, dont la «Chanson d'automne » qu'on analysera dans le présent article.

6 Notre article vise à démontrer la réception de l'harmonie verlainienne par Minsky. Pourtant, dans un premier temps, nous mettrons en avant la distinction de la pensée artistique de Minsky de l'auteur français. Grâce à ce point de départ, nous suivrons, à présent, le développement de la perception des nouveaux poèmes à leur réception et adaptation. Nous commencerons par les motifs verlainiens chez Minsky comme lecteur attentif et réceptif. Nous regarderons ses poèmes aux motifs musicaux et tenterons de confirmer qu'il s'appuyait sur l'expérience poétique de l'auteur français. Ensuite, nous rétrécirons notre explication jusqu'à l'analyse de la traduction du poème la «Chanson d'automne » faite par Minsky. Enfin, nous décrirons des mises en musique de cette traduction (1904) et présenterons la comparaison des indications musicales de deux compositeurs, Alexandre Kreïn et Emil Cooper. Nous essaierons ainsi de voir comment le rythme verbal est transposé en rythme musical; et dans un mouvement inverse, nous verrons comment la mise en musique affecte la perception du texte.

\section{Vision du monde de Minsky}

7 La littérature ne peut se développer sans réévaluation de l'expérience des prédécesseurs et des contemporains. Ainsi, l'œuvre de Verlaine est une source d'inspiration non seulement pour les symbolistes français mais aussi pour les auteurs russes. En Russie, on trouve la première mention de son nom dans l'article de Zinaida Venguerova «Les poètes symbolistes en France» (Vengerova, 1892: 115-143) publié dans Vestnik Evropy [Le Messager de l'Europe]. C'est la raison pour laquelle nous pouvons dire que Venguerova joue un rôle d'intermédiaire entre le symbolisme français et le russe. Grâce à elle, le symbolisme français attire l'attention des poètes russes.

8 L'intérêt de Nicolas Maksimovitch Minsky (1856-1937) - poète, dramaturge, philosophe, publiciste russe - pour la littérature française (francophone) devient 
évident à la lecture de ses œuvres comme des commentaires de ses contemporains. Par exemple, Brioussov écrit dans l'article "N. Minsky. Essai d'une caractérisation »: «Mais au début des années 1890 l'œuvre de Minsky a été marquée par un bouleversement brusque et fécond. Celui-ci a été provoqué, d'une part, par le travail intérieur de la pensée, d'autre part, sous l'influence de nouveaux écrivains européens. » (1975 : 237) Brioussov parle non seulement de l'importance des auteurs européens, mais aussi de la place de la réflexion philosophique dans l'élaboration des textes poétiques de Minsky. Zinaida Guippious écrit dans ses mémoires : «Quant aux écrivains novateurs français, ils étaient peu connus chez nous. [...] Seul Minsky récitait ses vers et s'efforçait de me passionner, mais il n'était pas capable de produire la moindre nouveauté. » (1943, consultation le 22 mai 2019) Nous pouvons déduire des paroles de Guippious, que Minsky s'intéressait lui-même aux auteurs français et qu'il tenait à les faire connaître au lecteur russe. Pourtant, Guippious met également l'accent sur un certain conservatisme de la poésie de Minsky, se référant, évidemment, à son œuvre précoce. En revanche, les chercheurs d'aujourd'hui ne relient presque pas le nom de Minsky aux symbolistes français. Il nous semble donc pertinent de chercher à comprendre dans quelle mesure cet auteur russe a été en rapport avec l'œuvre des poètes français, plus particulièrement avec l'œuvre de Verlaine. Pourtant, nous ne considérons Nicolas Minsky comme étant à la base de ce nouveau mouvement littéraire, que pour sa réception des poètes français. Ses propres raisonnements philosophiques ont poussé ses contemporains à la création d'une nouvelle poésie.

9 L'idée de Minsky selon laquelle chaque personne est vouée à la poussière, à l'obscurité, à la mort, au néant éternel devient un des principes majeurs du symbolisme précoce en Russie : «Je sais que la cause de mon néant ne se cache pas ailleurs que dans mon corps, dans mon cœur qui à cet instant même bat pour la joie de vivre et dans une heure, peut-être, fera courir un poison mortel dans mes veines.» (1897:25) Le sentiment de solitude absolue et d'abandon qui surgit à l'intérieur de sa «tour d'ivoire » et mène inévitablement à la fatigue, au dégoût pour la vie, au rêve de la mort comme une des formes de l'existence infinie distinguent Minsky de Verlaine. Les premiers symbolistes russes sont en quête d'une jouissance absolue, éternelle, qui ne se trouve pas dans la vie :

[...] Mais le plus immortel de tous est celui qui, à travers les cendres terrestres,

Croyait entrevoir au loin un nouvel univers,

Irréel et éternel,

Qui désirait tant le but invisible et qui souffrait tant,

Que sa soif violente a fait apparaître un mirage

Au milieu d'un désert infini ${ }^{4}$.

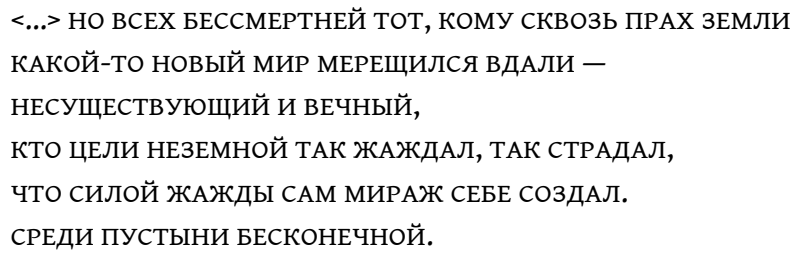

En même temps, selon Minsky, comme selon les symbolistes français, le poète est un translateur de la voix de l'Âme du monde, de cet inconnu. Autrement dit, l'art est perçu comme un principe fondamental de l'univers, dont le centre est la personne du poète. La poésie devient la force qui permet de distinguer deux idées proches : le néant et la mort. L'univers de Minsky se présente comme «le miroir de Dieu» dans lequel 
l'homme se reflète. Ce motif devient le symbole de la perte de soi-même à travers le conflit de deux abîmes, de deux vides.

11 Les motifs mortifères (pessimisme, scepticisme, individualisme extrême, etc.) provoquent le dégoût chez la majorité des lecteurs. Naturellement, l'écrivain se heurte à une critique cruelle et parfois injuste, fondée sur les normes de la morale. De plus, ses différentes tentatives de créer une nouvelle langue poétique ont parfois été vues comme une originalité superflue. Dans le monde russe, les œuvres de Minsky sont qualifiées du terme péjoratif de "décadentes ». Il est nécessaire de souligner que la notion du symbolisme littéraire n'est pas encore apparue. Nous en trouvons la confirmation, par exemple, dans les mémoires «Mes rencontres. Décadents» du critique Leonid Sabaneïev: "Pendant ces années, on les [les décadents] nommait toujours ainsi. Après c'était plus poliment "symbolistes". ( (Kreïd, 1993 : 343) Sabaneïev souligne que les contemporains renomment les nouveaux poètes au moment où leur point de vue envers eux change.

Comme un grand nombre de ses contemporains, il s'avère difficile de considérer Minsky comme un poète uniquement symboliste. Il a vécu une longue vie au cours de laquelle sa perception du monde a évolué. Les premières œuvres de Minsky sont apparues dans le champ littéraire en 1877 : son premier poème « Dans ma patrie » [ " HA РодинЕ »] a été publié dans la revue Vestnik Evropy [Le Messager de l'Europe]. Dans les années 1880, la poétique de Minsky se caractérise par une tonalité libérale-populiste, en réaction aux événements historiques: la "croisade vers le peuple ${ }^{5}$ " (fin 1873début 1874) de l'intelligentsia (des intellectuels) et la reconstitution du groupe populiste (ancien groupe révolutionnaire) Zemlia $i$ Volia [Terre et Liberté, 1875]. Schématiquement, le but de l'intelligentsia consiste à instruire les paysans et faire de la propagande révolutionnaire. Mais il voulait également suivre des tendances littéraires déjà existantes. S'il est difficile de classer l'œuvre de Minsky dans un mouvement littéraire particulier, la question des origines de ses positions esthétiques est tout aussi problématique. L'étape suivante de la voie littéraire de Minsky (vers 1886-1890) est caractérisée par des changements brusques du contenu de sa poésie ${ }^{6}$. À la place de la voix du peuple apparait la voix de l'auteur. Le poète est l'un des premiers en Russie à lever le drapeau de l'individualisme. Les motifs de la solitude, de la douleur, de la peur, de l'unité de la vie et de la mort sont la conséquence de l'indifférence du poète envers tout, sauf envers "soi-même». En même temps, l'auteur russe élabore sa propre conception philosophique du méonisme ${ }^{7}$, basée sur le rejet des autres «moi ». Cette étape est aussi marquée par l'adaptation et la réinterprétation des textes poétiques des auteurs français, surtout des œuvres de Verlaine. Ensuite, jusqu'en 1905, Minsky reconsidère sa théorie méonique, en la reliant à la religion. Mais en 1906, il est forcé de partir à l'étranger (Paris, Berlin, Londres). Après son départ, une « conspiration du silence " s'est formée en Russie autour de son nom. Par sa coopération avec les bolcheviks, le poète (ex-)symboliste a discrédité ses idéaux et a jeté une ombre sur le mouvement religieux de rénovation qui se développe alors en Russie. À partir de 1905, Minsky rencontre des difficultés pour publier ses œuvres (même lorsqu'il s'agit de la première période décadente de son œuvre). Pourtant, la cause de ces refus répétés n'est sans doute pas seulement sa collaboration avec les bolcheviks, ce sont ses textes et sa personnalité en général qui sont rejetés par ses contemporains russes. En guise de conclusion, indiquons qu'il est difficile de réunir les œuvres de Minsky autour d'un seul axe littéraire, comme l'œuvre de Verlaine. Généralement, la culture russe au tournant 
des $\mathrm{XIX}^{\mathrm{e}}-\mathrm{XX} \mathrm{e}^{\mathrm{e}}$ siècles se développait sous le signe d'une synthèse des arts, où la musique occupe une place centrale.

Il est important de mettre en avant la distinction de pensée artistique de Minsky pour ainsi déduire les motifs verlainiens chez l'auteur russe. Minsky, à la différence de Verlaine, s'enferme dans le monde de son « moi », s'isolant de la réalité qui provoque le mécontentement. Son bonheur se fonde sur la sensation de l'infini, de l'existence d'Idée et non pas de son corps. L'aspiration à saisir l'insaisissable pour que surgisse un éclat de l'Idée détermine ainsi l'atmosphère musicale de la poésie de Minsky. En même temps, dans le paragraphe suivant, nous tenterons d'expliquer l'autre type de musicalité qui unit les poètes.

\section{Motifs verlainiens chez Minsky}

L'œuvre de Minsky est un exemple de combinaison de différents genres et motifs, conjugués entre eux : dans la chanson, il y a le motif du hurlement du vent ou celui de la chute de feuilles; dans la prière, celui de la fatigue, de la déception et de la lueur d'espoir; dans les essais philosophiques, celui de la mélancolie. L'art est compris comme une révélation, une porte entrouverte sur l'Éternité. Parallèlement, Minsky, tout comme Verlaine, exige de son lecteur des efforts pour comprendre, imaginer et ressentir.

Ainsi, en 1901 paraitt le recueil de poésies de Nicolas Minsky Nouvelles chansons [новыE ПЕСНи], dont la sonorité et les motifs musicaux se manifestent non seulement dans le titre, mais aussi dans celui de certains de ses poèmes: «Le cantique des cantiques » [«ПЕСНЯ ПЕСЕН »]; «Chanson du soir» [« ВЕЧЕРНЯЯ ПЕСНЯ »]; «Chanson » [«ПЕСНЯ»]; «Elle se pencha au-dessus d'une harpe» [«НАД АРФОЮ ОНА СКЛОНИЛАСЬ »]; «Chanson d'automne » [« ОСЕНняЯ ПЕСНЯ »]; ou encore "Le bruit des feuilles (chanson d'automne)»[«ШЕЛЕСт ЛИСтЬЕВ (ОсЕНняЯ ПЕСНЯ)»], qui réapparait ensuite dans le cycle «Feuilles mortes» [« МЕРтвыЕ листья»]. Cette insistance de la musicalité dans les titres nous a fait penser à une retraduction de l'œuvre de Verlaine.

Sous le titre «Chanson d'automne » nous trouvons dans ce recueil deux poèmes, réunis par le même thème qui sont des allusions au poème homonyme de Verlaine: la première "Chanson d'automne" est apparue en 1896, puis «Le bruit des feuilles (chanson d'automne) » (1901). De plus, en 1904 dans ses œuvres complètes en 4 volumes (Minsky 1907), Minsky publie la traduction du texte poétique de Verlaine sous le titre "Chanson d'automne (de P. Verlaine) ». Cette chronologie montre que Minsky adapte le système de Verlaine, non seulement à sa traduction, mais aussi à sa propre interprétation. Rappelons que le premier livre de Verlaine (Romances sans paroles), intégralement traduit par Brioussov, a été publié en 1894, ce qui permet aux poètes de l'époque d'approfondir leur connaissance de la poétique verlainienne.

17 Commençons par l'analyse du poème adapté (daté de 1896 dans le recueil Novye pesni), dont le titre contient déjà une allusion au texte du poète français, de même qu'il reprend partiellement son sujet: les rêves anciens et les pleurs d'automne dans une âme malade. Des images impressionnistes caractéristiques de Minsky s'égrènent à partir de cette base : «La ville est drapée d'une robe d'automne » [ ГОРОД ЗАКУТАН В ОсЕНнИЕ РИЗЫ »] ; « La ville est drapée d'un brouillard bleu-gris » [« ГОРОД ЗАКУТАН В 
ТУМАН СВЕТЛО-СИЗЫЙ »] ; « Fumée immobile des feux éteints » [« ДЫМ НЕПОДВИЖНЫЙ потухших костров »], etc. De plus, grâce au rythme saccadé (intermittent) du dactyle (de trois ou quatre pieds) et aux assonances en o et $i$, l'auteur crée une structure sonore qui évoque une mélopée :

[...] À cette heure matinale ou tardive, incertaine

Des branches laissent tomber des feuilles sans bruit,

Le cœur enterre des rêves sans douleur

À cette heure pâle, douce et trouble. [...]

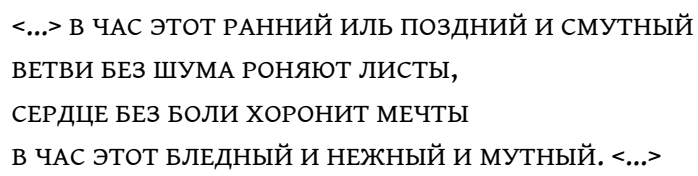

En outre, au niveau lexical l'auteur souligne que le hurlement est étouffé : sans bruit («БЕЗ ШУМА ), les couleurs fades donnent une impression diffuse au texte: pâle (« БлЕдный »), trouble (« мутный »). Pour mieux évoquer l'état d'âme, Minsky utilise les mêmes couleurs que Verlaine, ce qui permet de créer des images pleines de mélancolie. Il rend visibles son découragement, ses états d'âme, sa tristesse aiguë, à l'aide de demi-teintes et de couleurs douces. Même dans les poèmes ultérieurs ${ }^{8}$, le héros lyrique de Minsky est presque toujours dans l'ombre, les poésies sont dépourvues de couleurs vives: "Soudain dans ma prison muette, / Dans une nuit épouvantable » («Un lit ») [«ВДРУГ В НЕМОЙ МОЕЙ ТЮРЬМЕ, / В ЖУТКОЙ ТЬМЕ» («ПОСТЕЛЬ »)]; «Partout où il fait silence, nuit et solitude, / Où la vie s'en va, tu apparais, / Comme un écho du silence, comme une ombre du noir » («Peur») [«ВЕЗДЕ, ГДЕ БЕЗМОЛВНО, ТЕМНО, ОДИНОКО, / ГДЕ ЖИЗНЬ УБЫВАЕТ, ЯВЛЯЕШЬСЯ ТЫ, / КАК ЭХО МОЛЧАНЬЯ, КАК ТЕНЬ ТЕМНОТЫ » (« СТРАХ $)]$;

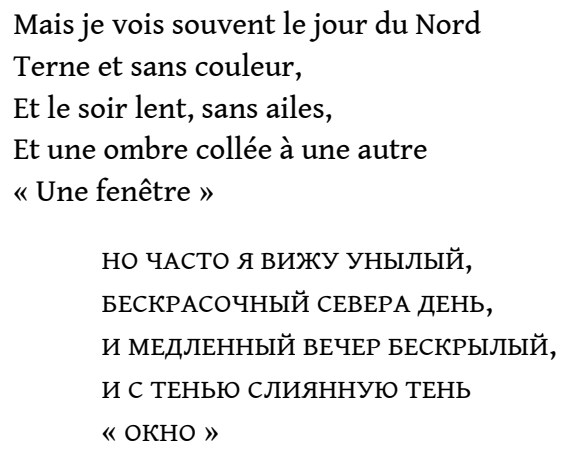

Dans le poème "Une fenêtre ", pour approfondir les émotions du héros lyrique, l'auteur use du barbarisme sliyannyï [«слиянный »]: « collée à une autre» comme pour accentuer la profondeur de l'ombre. Cette harmonie du poème permet au lecteur de songer à la solitude profonde éprouvée par le locuteur. Le sentiment d'abandon qui le saisit à l'intérieur de sa "tour d'ivoire ", le mène à l'épuisement, suscite le dégoût de la vie et le fait rêver à la mort, vue comme une des formes de l'existence infinie. La mort est perçue comme faisant partie de la vie du poète. Ces spécificités différencient Minsky des fondateurs du symbolisme français, mais ils sont typiques de la poésie décadente. Minsky accentue délibérément les traits de cette perception décadente du monde et traduit les motifs apocalyptiques.

C'est aussi, bien sûr, ce que suggère l'automne, qui n'évoque pas seulement la mort de tout le vivant, mais aussi le caractère cyclique de la vie. La mort est donc une étape 
passagère qui mène à une vie nouvelle. Ainsi, la vie apparait sans bruit et son caractère cyclique ininterrompu se manifeste nettement dans le poème suivant, «Le bruit des feuilles (chanson d'automne) ». Avec «Le repos » arrive une nouvelle vie, une nouvelle saison de l'année :

Par une nuit d'orage ou par un midi froid Nous sommes seuls, notre mort en beauté transformée, Nous nous détacherons des branches, légères et libres, Nous nous envolerons, chuchotant et brillant.

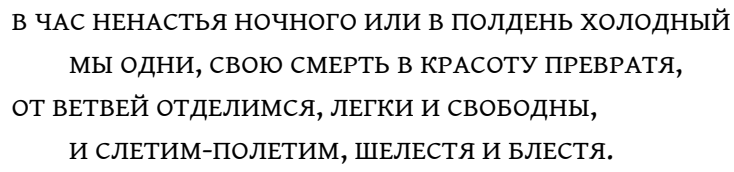

21 La mort métaphorique des feuilles et leur «brillance » (l'image de la vie après la mort) montrent la boucle de la vie créatrice. Ceci est également souligné grâce à la variation de construction des vers. Pour faire chanter la nature, Minsky croise des anapestes de cinq et quatre pieds aux rimes pauvres. En outre, la dernière strophe est riche en combinaisons de consonnes qui interagissent entre elles : $\mathrm{sl} / \mathrm{t} / \mathrm{mp} / \mathrm{l} / \mathrm{mch} / \mathrm{l} / \mathrm{st}$ / bl / st, pour évoquer les sonorités des feuilles qui s'envolent. Minsky fait l'échange harmonique entre l'expression et l'impression.

La poétique de Verlaine est nettement transmise par Minsky. La fatigue du héros lyrique prend des tournures mélancoliques. En même temps, ils préfèrent écrire à la première personne du singulier pour projeter les émotions du poète, ce qui leur permet de faire une correspondance entre le paysage intérieur (leurs âmes) et extérieur. Les deux auteurs évoquent ainsi la nature et sa musicalité. Minsky, comme Verlaine, parle de la fuite du temps avec laquelle le héros lyrique s'efface derrière la nature. Il reproduit la métaphore de l'âme du poète, à la fois lyrique et musicale.

Grâce au poème «Chanson d'automne » de Verlaine, est né dans l'œuvre du poète russe un cycle propre, unifié par les motifs mélancoliques de l'automne. La saison grise et sombre de Minsky change de couleurs; le lecteur voit le passage de l'obscurité à la lumière : au début nous lisons « Il n'y avait ni soleil, ni azur, ni lointain... / Pas de vie, ni d'enterrement» ("Chanson d'automne», 1896) [«НЕ БЫЛО СолНЦА, ЛАзУРИ И ДАЛИ... / НЕ БЫЛО ЖИЗНИ, И НЕТ ПОХОРОН » (« ОСЕННЯЯ ПЕСНЯ »)], et ensuite « Les branches flambent aux feuilles jaunies / Sur les bouleaux doux l'aube rosit» («Automne», 1902) [«ТО ВЕТВИ ПЫЛАЛИ ПОЖЕЛТЕЛОЙ ЛИСТВОЙ./ НА НЕЖНЫХ БЕРЕЗАХ РОЗОВЕЛА ЗАРЯ...» («ОСЕНЬ )). Dans le paragraphe suivant nous verrons comment Minsky passe d'un cycle poétique inspiré par Verlaine à la traduction de ses œuvres, notamment «Chanson d'automne ».

\subsection{Minsky traducteur de Verlaine}

L'étude de la traduction du poème "Chanson d'automne" permettra de mettre en lumière ce qui unit les poétiques de Verlaine et de Minsky. Dans son dernier recueil De l'obscurité vers la lumière (Minsky, 1922) dans lequel les textes sont organisés par thèmes, ce poème est classé parmi les textes lyriques « désespérés ». Par ce classement, Minsky souligne son état d'esprit. Déjà, dans la première strophe, grâce au choix lexical, nous entendons le gémissement mélodieux d'une âme malade, les mots « résonnent comme une corde » (« звучит струной »): 


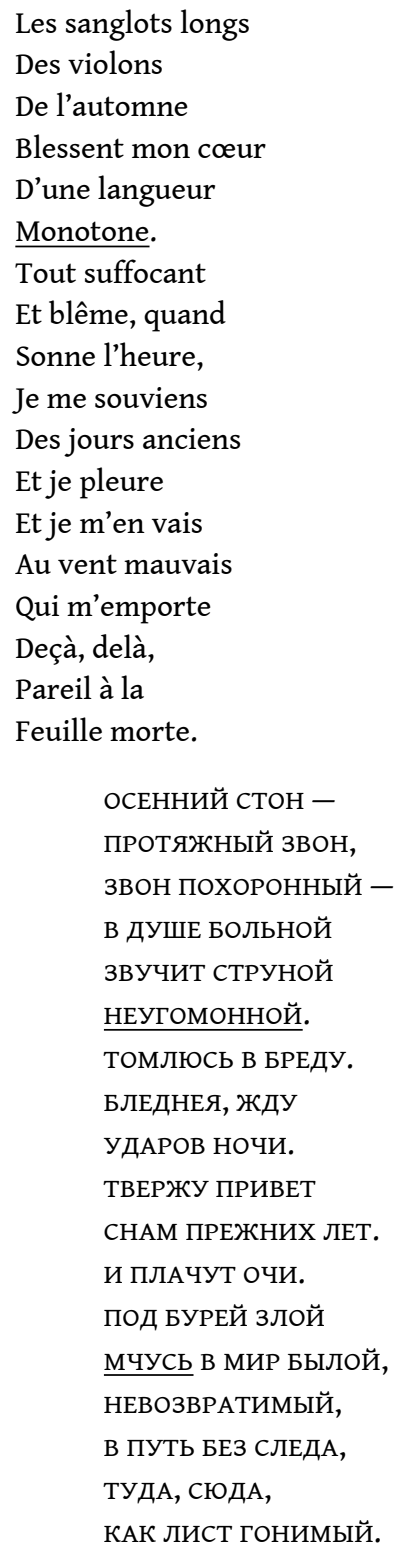

Nous pouvons remarquer tout d'abord que Minsky essaye de garder le vers de quatre syllabes de Verlaine et son type de rime (aabccb) pour transmettre le rythme d'une chanson. Néanmoins, les terminaisons des vers ne sont pas toujours respectées. L'ïambe (presque toujours) de deux pieds n'est pas loin de la structure rythmique du texte français. Le niveau lexical, lui aussi, est très proche, excepté pour deux mots: neugomonnyï [«НЕугОмонной»] et mtchus [« Мчусь»]. L'adjectif neugomonnyï (inlassable) exprime un mouvement continu, tandis que "monotone» en français signifie qu'il s'agit du même ton ou un ton peu varié. Quant au verbe mtchus (je me précipite), il est très diffèrent de "s'en aller ». Le fait de se lancer ou même de courir rapidement est plus rapide et brusque qu'un seul changement du lieu (" je m'en vais »). Du point de vue du sens, Minsky ajoute donc de l'action. Notons que Verlaine évoque la musique dans son texte à travers l'utilisation du verbe sonner et à la référence aux violons, tandis que Minsky remplace le verbe par le nom udar (coup). Les coups de nuit de Minsky font référence ainsi à l'horloge qui sonne dans la nuit. Les violons également sonnent dans l'âme du héros lyrique. 

sont musicales. Pour insister sur la monotonie, les deux poètes ont recours à l'allitération en " 1 / л », répétitive, suggestive, comme pour faire référence au son de violon et éveiller les sentiments de la douleur et de la tristesse. La suggestion chez Minsky et Verlaine s'explique par le fait qu'ils veulent éveiller les émotions profondes des lecteurs pour mieux transmettre leurs sensations. Par le principe de suggestion, ils ne décrivent pas le monde mais traduisent en série de nuances les impressions que le monde fait naître. Il paraît approprié de citer Mallarmé qui affirme «peindre non la chose, mais l'effet qu'elle produit» (Bellet, 1993: 70). Minsky comme Verlaine s'écartent d'une norme littéraire stricte, et mêlent des éléments visuels et auditifs où le mot, devenu musical, sert à exprimer l'humeur. Nous pouvons également remarquer une évolution en ce qui concerne la prosodie des poèmes de Minsky: les mots polysyllabiques qui ralentissaient le rythme laissent place à des phrases plus laconiques.

Les motifs mélancoliques et le caractère impressionniste de l'œuvre de Verlaine deviennent un des principes de la poésie de Minsky. Pourtant, le dessin rythmique des œuvres de Minsky se distingue de celles de Verlaine. Grâce aux répétitions sonores, aux intonations mélodieuses, aux assonances, Verlaine, en quête de spiritualité, transformait la poésie en musique, tandis que Minsky brise l'intonation et la mélodie de ses poèmes ultérieurs, expérimente le vers libre, tente de traduire la fracture de son âme, sa fatigue mortelle.

La richesse poétique de la "Chanson d'automne » consiste en sa simplicité qui atteste ainsi de la modernité du poète. Les codes de la poésie romantique sont dépassés par la forme musicale et le dévoilement de l'âme triste et fatiguée. Le poète aspire à créer une nouvelle langue poétique pour transmettre un autre état d'esprit et une autre perception du monde. La brièveté du texte facilite une mise en musique éventuelle. Dans le paragraphe suivant, nous montrerons l'intérêt des musiciens pour la traduction de Minsky.

\subsection{Mise en musique de la traduction de Verlaine faite par Minsky}

Dans le fonds des notes et enregistrements sonores de la Bibliothèque nationale de Russie, nous trouvons un intérêt constant aux œuvres de Minsky des musiciens de différentes époques, passionnés par les romances: Rachmaninov - «Dans mon âme l'amour se lève...» [« В мОЕЙ дУшЕ люБовь восходит... »], «Elle est belle comme midi...» [« ОНА КАК ПОЛДЕНЬ ХОРОША...»]; Glière - «Chanson orientale " [« ВосточнАЯ ПЕСНЬ »]; une sérénade de Davingof - « De lourds nuages traînent dans le ciel... » [« ТЯНУТСЯ ПО НЕБУ ТУчИ ТЯЖЕЛЫЕ...»]; Zolotaryov (1942-1975) — «La vague » [« ВОЛНА »], «À l'aube » [« ПЕРЕД ЗАРЕЮ »]; Mouromtsevsky - la romance «Elle m'attire... » [« ОНА МЕНЯ влЕчЕТ... »], la valse-romance «Pardonne et oublie » [« ПРостИ И ЗАБУДЬ »]; Rozenov - « Je l'ai vue » « Я УВИДЕЛ ЕЕ »], « Trois Parques » [« ТРИ ПАРКИ »], « Clair de lune » [« лУннЫЙ СВЕТ »], « Quand dans l'âme froide le soir vient... » [« КОГДА В ДУШЕ ОСТЫВШЕЙ ВЕЧЕРЕЕТ... »], « Je n'aime pas encore » [« ЕЩЕ Я НЕ люБлю »], « Chanson orientale » [« Восточная ПЕСНь »], etc. Cet intérêt pour les œuvres du poète au début des années 1900 est sans doute dû en grande partie à leur dimension musicale. Cette énumération pourrait également élargir à une autre facette

ILCEA, 38 | 2020 
de la question comment on "chante" les poètes. En revanche, concentrons-nous, à présent, sur la mise en musique du texte de Verlaine traduite par Minsky.

La traduction russe de "Chanson d'automne » [« ОСЕНнЯя ПЕСНЯ », 1904] de Verlaine faite par Minsky a été mise en musique par Alexandre Kreïn (1909) et par Emil Cooper (1914), dont nous avons trouvé trace dans le fonds des notes et enregistrements sonores de la Bibliothèque nationale de Russie. Dans les partitions de Kreïn, nous ne trouvons que le texte russe, et dans celles de Cooper le texte est disponible en trois langues : en français, en russe et en allemand (traduction de Lina Esbeer). Cooper, qui préférait pourtant les traductions de Verlaine faites par Brioussov', a adapté la "Chanson d'automne", traduite par Minsky, pour soprano et piano. Comparons les indications musicales des deux compositeurs afin de comprendre comment elle influence la perception du texte.

31 Les deux compositeurs commencent lentement, sans hâte, Cooper précise "Andante tristemente »-mouvement doux et triste. Le rythme se maintient jusqu'au septième vers, la monotonie des mots favorise un tel accompagnement musical. À partir du huitième vers, «l'inquiétude» (« inquieto », Cooper) monte, la musique devient un peu plus vive («poco più mosso», Kreïn). La répétition d'actes privés de sens provoque l'angoisse: «le délire» (БРЕД), suscite des images surgissant à leur gré dans la conscience et exprimant un mal-être; le mot "coups" (удАРы) sonne comme le carillon d'une horloge, creux et inattendu. De même, le verbe « insister » (ТВЕРдить), créent la même impression et l'adjectif « ancien » (ПРЕЖнИх) évoque le retour à ce qui a déjà eu lieu. Chez Cooper, les vers dix, onze et douze résonnent deux fois, comme un couplet : il a perçu la répétition des actions. Au vers treize, l'inquiétude diminue chez les deux compositeurs, il y a plus de modération, de retenue. Durant les six derniers vers, les musiciens reviennent au tempo originel, en ralentissant le rythme de la musique vers la fin. Il convient de noter que les compositeurs divisent le poème en trois parties et que le rythme dépend avant tout du niveau lexical.

Les images créées par la musique du texte dépendent non seulement du niveau de perception et de culture du lecteur mais aussi de la reproduction musicale. Les poètes mettent en valeur le caractère ambivalent de l'énergie langagière, tandis que les musiciens font la réception du texte claire et monodirectionnelle.

\section{Conclusion}

En conclusion, nous pouvons noter que les mélodies mélancoliques et l'impressionnisme de la poésie verlainienne inspirent le poète russe. Essentiellement, Minsky fait une adaptation ou une réinterprétation des textes de Verlaine. Cette adaptation peut être divisée en deux types : l'emprunt d'un titre qui a suggéré l'idée d'un nouveau poème ayant la même image et le même dessin mélodique ; l'emprunt de l'idée générale d'une œuvre qui a passionné Minsky et qui entre en résonance avec celle du poète. L'harmonie des poèmes de Minsky consiste en ce qu'il tente, après Verlaine, de transmettre l'humeur et le rythme de ses émotions où leurs sentiments sont inévitablement liés à la notion de fatigue. Nous observons donc des tentatives hésitantes de Minsky de la réalisation du principe symboliste selon lequel les buts d'un art sont atteints par les moyens d'un autre. 

poète, qui lui a permis de prendre connaissance de l'expérience des auteurs étrangers, de même que de mieux se connaître, de faire épanouir son talent. Pour Minsky, le travail sur la traduction a permis de toucher à la «musique des sphères » de Verlaine. En aiguisant systématiquement son oreille, il apprenait à voir les sons et à entendre les couleurs pour profiter de toute la richesse de la vie. L'accompagnement musical de "Chanson d'automne" permet de faire remonter la profondeur des sensations à la surface de la conscience.

Ainsi notre mise en avant de la réception de l'harmonie verlainienne par Minsky nous a permis de mettre en évidence comment le rythme verbal est transposé en rythme musical; et en même temps nous avons souligné comment la mise en musique influence la perception du texte.

\section{BIBLIOGRAPHIE}

BELLET Roger (1993), Stéphane Mallarmé : l'encre et le ciel, Seyssel : Champ vallon.

BRIoussov Valery (1975), « N. Minsky. Essai d'une caractérisation. 1908 » [« N. Minsky. Opyt harakteristiki. 1908 », Brioussov, Euvres complètes [Sobranie sočinenij, vol. 6], Moscou :

Hudožestvennaja literatura, 235-241.

COOPER Emil (1914), Chant d'automne : pour le soprano avec piano [Osennjaja pesnja: Dlja vysokogo golosa $s$ fortepiano, Op. $\left.8, n^{\circ} 1\right]$, Moscou : Fonds des notes et enregistrements sonores de la Bibliothèque nationale de Russie.

GuIPPIous Zinaida (1943), « Dimitri Merejkovski » [« Dmitrij Merežkovskij »], <http://az.lib.ru/g/ gippius_z_n/text_1943_merezhkovsky.shtml> (consulté le 22 mai 2019).

HANSEN-LÖVE Aage (1999), Symbolisme russe. Système des motifs poétiques. Le symbolisme précoce [Russkij simvolizm. Sistema poètičeskih motivov. Rannij simvolizm], Saint-Pétersbourg: Gumanitarnoe Agentstvo « Akademičeskij Proekt ».

KARACUBA Irina Vladimirovna, KURUKIN Igor Vladimirovič \& SOKOLov Nikita Pavlovič (2015), Choisir son histoire. Les bifurcations sur le chemin de la Russie : des Rurikides aux oligarques [Vybiraja svoju istoriju. Razvilki na puti Rossii: ot Rjurikovičej do oligarhov], Moscou : AST, Corpus.

Kreïn Alexandre (1909), "Chant d'automne » : Pour le chant avec piano : h-a. 2 : Op. 4, $n^{\circ} 1$, Moscou : Fonds des notes et enregistrements sonores de la Bibliothèque nationale de Russie.

KREÏD Vadim (1993), Mémoires de l'Âge d'argent [Vospominanija o serebrjanom veke], Moscou : Respublika.

LESOURD Françoise \& MASLINE Mikhaïl (2010), Dictionnaire de la philosophie russe, Lausanne : L’Âge d'Homme.

MiNSKY Nicolas (1888), Poèmes [Stihotvorenija, 2 éd], Saint-Pétersbourg : Tip. V. S. Balaševa. 
MINSKY Nicolas (1897), À la lumière de la conscience. Pensées et rêveries sur le but de la vie [Pri svete sovesti. Mysli i mečty o celi žizni], Saint-Pétersbourg : Tip. Û. N. Èrlih.

MinSKY Nicolas (1907), CEuvres complètes en 4 volumes [Polnoe sobranie stihotvorenij $v 4$ tomah], SaintPétersbourg : M. V. Pirožkov.

MORÉAS Jean (1886), « Un Manifeste littéraire », Le Figaro, 18 septembre 1886.

VENGERoVA Zinaida (1892), « Les poètes symbolistes en France » [ Poèty simvolisty vo Francii »], Vestnik Evropy, 9, 115-143.

VerLAINE Paule (1894), Romances sans paroles [Romansy bez slov, Valery Brioussov, trad.], Moscou : Tip. È. Lissnera i Ju. Romana.

VERLAINE Paule (1907), CEuvres complètes. Poèmes saturniens. Fêtes galantes. Bonne chanson. Romances sans paroles. Sagesse. Jadis et naguère (t. $1.5^{\mathrm{e}}$ éd.), Paris : Librairie Léon Vanier.

\section{NOTES}

1. L'année 1857, où paraît Les Fleurs du mal de Baudelaire, est à l'origine de l'apparition d'une nouvelle poésie. Les bases d'un nouveau mouvement littéraire sont posées dans l'œuvre de Rimbaud et de Verlaine dans les années 1871-1873. À partir de 1877 les nouveaux poètes se réunissent dans le salon de Mallarmé. La formation aboutit à la publication de Un Manifeste littéraire de Jean Moréas en 1886 où les nouveaux poètes sont nommés «symbolistes ». Dans l'article présent, nous considérons le début des années 1870 comme la date de la naissance du symbolisme en France.

2. Soulignons que la division du symbolisme russe en deux étapes est la plus courante: le symbolisme " aîné » ou bien « la décadence » à partir des années 1890 et le symbolisme " cadet » qui apparaît au début des années 1900. Autrement dit, nous parlons de deux vagues du symbolisme russe où la première vague des années 1890 commence le nouveau mouvement littéraire.

3. Minsky fait publier ces traductions de Verlaine dans la revue Novyï Put [La nouvelle voie] dans les années 1902-1904.

4. Les traductions en français sont celles de l'auteur. Ce poème « Comme un rêve, les actes et les désirs humains vont s'écouler... » a été publié dans sans recueil Stihotvorenija (Minsky, 1888).

5. Voir également à ce propos Karacuba, Kurukin \& Sokolov (2015 : 345-376).

6. En Russie la montée révolutionnaire des années 1870 , suivie d'une réaction profonde, la déception après l'abaissement de la vague révolutionnaire, les nouvelles recherches ont provoqué le besoin d'impératifs poétiques, de réflexions lyriques, de confessions. Les écrivains d'une «nouvelle conscience» définissent la période entre les années 1880 et 1890 , dite du «présymbolisme» d'après la définition du slaviste autrichien Aage Hansen-Löve (1999), non comme un courant ou une école littéraire mais comme le moyen d'atteindre la connaissance primordiale du monde.

7. Cette traduction convaincante du terme "mèonizm» a été trouvée dans Dictionnaire de la philosophie russe (Lesourd \& Masline, $2010: 563$ ).

8. Les poèmes sont publiés dans CEuvres complètes en 4 volumes de Minsky (1907).

9. Dans les archives de Cooper, nous remarquons qu'il a essentiellement recours aux traductions de Brioussov. 


\section{RÉSUMÉS}

L'œuvre de Verlaine n'est pas seulement une source d'inspiration pour les symbolistes français. En Russie, ses poèmes sont le plus souvent traduits par Valéry Brioussov et Fiodor Sologoub. Ils sont également traduits par Nicolas Maksimovitch Minsky (1856-1937) - poète, dramaturge, philosophe, journaliste russe. Il publie en 1901 son recueil de poésies Nouvelles chansons; on y trouve sous le titre "Chanson d'automne" deux poèmes, réunis par le même thème. Dans le recueil suivant (1904), Minsky publie la traduction du texte poétique de Verlaine sous le titre «Chanson d'automne (de P. Verlaine) ». Dans cet article, nous mettons en lumière l'adaptation que fait Minsky du système poétique de Verlaine. Ensuite nous analysons la traduction de l'œuvre de Verlaine par Minsky. Nous analysons également la notation musicale de ce texte russe, composée par les compositeurs Alexander Kreïn (1909) et Emil Cooper (1914).

Verlaine's work is not only a source of inspiration for the French symbolists. In Russia, his poems are most often translated by Valery Bryusov and Fyodor Sologub. However, Nicolas Maksimovich Minsky (1856-1937)-poet, playwright, philosopher, Russian publicist-also translated some poems. In 1901, his collection of poems New songs appears; we find there, under the title "Chanson d'automne" two poems united by the same theme. In the following collection (1904), Minsky publishes the translation of Verlaine's poetic text under the title "Chanson d'automne (by P. Verlaine)". In this paper, we highlight the analysis of the translation of Verlaine's work by Minsky. We also analyze the musical notation of this Russian text, compiled by the composers Alexander Kreïn (1909) and Emil Cooper (1914).

\section{INDEX}

Mots-clés : poésies russe et française (fin du XIXe siècle - début du XXe siècle), symbolisme (mouvement littéraire), Paul Verlaine, Nicolas Minsky, harmonie, poème « Chanson d'automne ", Alexander Kreïn, Emil Cooper

Keywords : Russian and French poetry (the late 19th century - early 20th century), Symbolism (literary movement), Paul Verlaine, Nicolas Minsky, harmony, poem "Chanson d'automne", Alexander Krein, Emil Cooper

\section{AUTEUR}

\section{DARIA KUNTSEVICH}

Université Bordeaux Montaigne

d.kuntsevich@gmail.com 